\title{
Object-motion sensitivity loss due to motion in the peripheral visual field
}

\author{
JAMES C. MUNDT \\ University of Wisconsin-Madison, Madison, Wisconsin
}

(Leonard E. Ross, Sponsor)

\begin{abstract}
The effect of peripheral-field movement on detection of object motion was investigated in two experiments. In each experiment, 24 subjects judged the movement of targets while elements in the subject's peripheral visual field were moving and while they were stationary. Targets were stationary or were moving at speeds between 6.2 and 23.1 minutes of visual arc per second. Target locations were to the left or right of (Experiment 1), or above or below (Experiment 2), a central fixation stimulus. Peripheral-field condition, direction of target movement, and speed of target movement significantly affected motion detection. The interaction between direction of target movement and speed of target movement was found to be significant in the analyses of both experiments. Target location was found to be a significant factor in Experiment 2, and sensitivity differences were found between horizontal and vertical target movement. The decrease in sensitivity due to peripheral-field motion did not depend upon subjects' experiencing vection.
\end{abstract}

Interaction between the visual and vestibular sensory systems has been, and continues to be, an important research topic. Input from the peripheral visual field is important for spatial orientation and the sensing of passive body movement (e.g., Held, Dichgans, \& Bauer, 1975; Lishman \& Lee, 1973), and vestibular efference has been found to affect a variety of visual functions, such as accommodation (Clark, Randle, \& Stewart, 1977), the vestibuloocular response (Jones, 1982), and nystagmus (Holm-Jensen, 1982).

Object-motion detection is also affected by vestibular influences. Reaction times to the onset of motion of a target have been shown to be significantly longer during angular head displacements than when the head is fixed in space (Büchele, Degner, \& Brandt, 1980; Probst \& Wist, 1982). In addition, both forward movement in a vehicle and peripheral-field stimulation inducing linear vection have been found to result in longer reaction times to size changes of an ellipse (Probst, Krafczyk, Brandt, \& Wist, 1984).

In the present experiments subjects were asked to judge whether targets were stationary or moving when stimuli in the peripheral visual field were in linear motion, which was intended to induce vection, and when they were stationary. In Experiment 1 target locations were $7.5^{\circ}$ to the left or right of a fixation stimulus that was at eye level in the sagittal plane, and in Experiment 2 target locations were at the same eccentricity above or below this point.

This research was supported by Grant AA06093 from the National Institute on Alcohol Abuse and Alcoholism. The author would like to express his appreciation to Leonard Ross for his patience and encouragement during this project, and to Hona Lee Harrington for her assistance in collecting data. The author's address is Department of Psychology, University of Wisconsin, Madison, WI 53706.
It was predicted that movement in the peripheral field would decrease sensitivity to target movement and that the effects of peripheral-field movement on the detection of object motion would interact with the direction of movement and target location due to sensory input expectations.

\section{METHOD}

\section{Subjects}

Separate groups of 24 students served as subjects in each experiment. Their ages ranged from 17 to 34 years. Subjects were required to have natural or contact-lens-corrected vision of at least 20/30 near and far vision in both eyes as measured by a Model OV-7 Titmus Vision Tester. The data of 15 additional subjects were not analyzed. Of these, 13 were not able to meet fixation requirements, as described below, and the other 2 were rejected on the basis of post hoc analyses of their data that indicated flagrant failure to comply with instructions.

\section{Apparatus and Stimuli}

Two raster scan cathode-ray tubes (CRTs), measuring $58.4 \mathrm{~cm}$ diagonally and facing one another at a distance of $76 \mathrm{~cm}$, were used to present peripheral-field stimulation. The subject was seated between the CRTs so that each screen stimulated the periphery from $37^{\circ}$ to $90^{\circ} \mathrm{ec}$ centricity. The stimulus on the side screens was controlled by a computer, with the horizontal deflection yoke reversed on one of the CRTs so that the two CRTs produced mirror-image patterns. The stimulus on the side screens consisted of a random pattern of luminous rectangles that were $1 \mathrm{~cm}$ in width and $0.5 \mathrm{~cm}$ in height. The rectangles had a luminous intensity of 0.5 nit and, on average, occupied $40 \%$ of the screen. When the rectangles were moving, they moved smoothly from the front edge of the screen to the back at a speed of approximately $1 \mathrm{~m} / \mathrm{sec}$.

A Model 1321A Hewlett-Packard $X Y$ monitor (P15 phosphor), positioned $70 \mathrm{~cm}$ directly in front of the subject, was used to present target stimuli. Two black panels with $34 \mathrm{~cm}$ (horizontal) $\times 25.5 \mathrm{~cm}$ (vertical) openings, situated $2 \mathrm{~cm}$ and $33 \mathrm{~cm}$ in front of the monitor, prevented the side CRT screens from illuminating the face of the monitor.

The central fixation stimulus and the targets to be judged were 0.74mm luminous squares, presented $7.5^{\circ}$ to the left or right (Experiment 1) or $7.5^{\circ}$ above or below (Experiment 2) the subject's forward horizon- 
tal axis. The luminous intensity of the targets was 0.04 nit in Experiment 1 and 1.94 nits in Experiment 2. Each target was presented for $2 \mathrm{sec}$, during which time it was either stationary or moving at 6.2, 9.7, $15.1,19.5$, or 23.1 minutes of visual arc per second to the left, right, up, or down. Presentation of the target stimuli was controlled by computer by means of a Tecmar digital-to-analog (D/A) converter board.

A Model 200 Narco Bio Systems infrared limbus eye-tracking device measured horizontal and vertical eye position. Eye position was sampled by computer, as were the buttonpress responses of the subject that indicated whether or not target movement had been detected.

\section{Design and Procedure}

Each experimental session consisted of two blocks of 128 trials each, with a 5-min break between trial blocks to minimize eye fatigue. Each trial block was broken into four sets of 32 trials. The trial sets within each block of trials alternated between stationary and moving peripheralfield conditions, with one block beginning with the peripheral field in motion, and the other block beginning with the peripheral field stationary. Presentation order of the trial blocks was balanced across subjects.

The subject was seated in an ophthalmological examination chair, with his/her head stabilized by a chin- and headrest. After the subjects received instructions, the lights in the experimental room were turned off and the eye-tracking glasses were calibrated. The subjects were given two sets of practice trials, five trials with the peripheral stimulus in motion and five with the peripheral visual field stationary.

For $1 \mathrm{~min}$ prior to each set of trials, a stationary fixation stimulus was presented with the peripheral-field stimulation to be used for that trial set present. The 32 trials within each trial set were balanced between the two possible target locations in a pseudorandomized order such that the same location did not occur more than three times in a row. Of the 16 trials at each of the locations within a set, 6 presented stationary targets and the remaining 10 involved two occurrences of targets moving at each of the five movement speeds. The four movement directions were balanced within each trial set. The central fixation stimulus was presented for a random interval of 2.5-3.5 sec at the beginning of each trial.

If fixation was not within $1^{\circ}$ of the calibrated zero point immediately prior to each trial, the computer suspended the trial sequence to permit calibration of the eye-tracking glasses. Due to the disruptive nature of the calibration procedure, the data of subjects who required more than 40 calibration checks during the experiment were not included in the study. If fixation was within the $1^{\circ}$ criterion the target to be judged was presented, and saccadic latency was determined by the computer as the time between target onset and the first eye-position sample outside the fixation criterion in the direction of the presented target. As soon as an eye movement toward the targets was detected, movement of the target and timing of the presentation interval began. Subjects were required to make a target movement judgment before the next central fixation stimulus was presented.

\section{RESULTS AND DISCUSSION}

In each of the experiments the subjects received 256 trials. Of these, 160 involved moving targets ( 2 trials for each of 2 peripheral-field conditions, 2 target locations, 4 movement directions, and 5 movement speeds), and 96 involved stationary targets (24 trials for each of 2 peripheral-field conditions and 2 target locations). The primary data for both stationary- and moving-target trials were the number of responses indicating that the subject had detected movement.

\section{Stationary Targets}

In both experiments, stationary targets were judged to be moving significantly more often when the peripheral stimulus was moving than when it was stationary. In Ex- periment 1 , stationary targets were judged as moving $40 \%$ of the time when the peripheral stimuli were moving and $24 \%$ of the time when they were stationary $[F(1,23)=$ $35.31, p<.001]$. In Experiment 2, 33\% of the stationary targets were judged as moving during the moving peripheral-field condition, compared with $22 \%$ during the stationary peripheral-field condition $[F(1,23)=17.44$, $p<.001]$. Clearly, the "stability" of stationary targets was affected by movement in the peripheral visual field.

The only other significant main effect in the responses to stationary targets was found in Experiment 2, where the average number of incorrect judgments of stationary targets at the top location (21\%) was significantly less than the number made of targets at the bottom location (34\%) $[F(1,23)=25.89, p<.001]$.

\section{Moving Targets}

The ability to detect target movement when it did occur was also affected by the peripheral-field viewing condition in both experiments. In Experiment 1, subjects correctly judged $74 \%$ of the moving targets when the peripheral-field stimuli were stationary, compared with $66 \%$ when they were moving $[F(1,23)=40.93$, $p<.001]$. The corresponding percentages of correct judgments of moving targets in Experiment 2 were $66 \%$ and $59 \%[F(1,23)=7.68, p<.05]$. These differences were undoubtedly less than they might have been due to the range of target speeds used. Near chance detection rates were found for the slowest two target speeds for both peripheral-field conditions, indicating a floor effect for those speeds.

A description of the effect of peripheral-field motion should jointly consider judgments of moving and stationary targets. Consequently, a regression analysis of $d^{\prime}$ measures collapsed over subjects (Macmillian \& Kaplan, 1985; Swets \& Pickett, 1982) was conducted, which revealed that the peripheral-field manipulation accounted for $31 \%$ of the total variance in Experiment 1 and $18 \%$ in Experiment 2 (both $p s<.001$ ).

As expected, the likelihood of a correct response to a moving target increased with increasing target speed in both experiments $[F(4,92)=88.10$ and 90.89 in Experiments 1 and 2, respectively; both $p s<.001$ ].

The direction of movement also had a significant effect in both experiments $[F(3,69)=16.78$ and 23.91 for Experiments 1 and 2, respectively; both $p s<.001]$. The nature of the effect was not the same in each experiment, however. A Newman-Keuls post hoc comparison of the mean number of correct responses $(p<.05)$ indicated that upward target movements were more likely to be correctly identified than were movements in the other directions in Experiment 1, whereas downward movements were less likely to be identified than were movements in the other directions in Experiment 2.

The interaction between speed of movement and peripheral-field viewing condition was also significant in both experiments $[F(4,92)=8.39, p<.001$, and 4.86 , $p<.01$, for Experiments 1 and 2, respectively]. This 
interaction reflects the fact that the peripheral-field condition did not influence movement detection rates at the slowest movement speed, but did affect detection rates at the three fastest speeds.

The speed of target movement $\times$ direction of movement interaction was significant in both experiments $[F(12,276)=2.34, p<.01$, and $F(12,276)=3.46$, $p<.001$, for Experiments 1 and 2, respectively]. In both experiments, the detection rates of movements to the left or right increased in a parallel manner as target speed increased, but diverged from the detection rates of upward and downward target movements, which were also parallel as target speed increased. Probst et al. (1984) also found significant response differences to vertical and horizontal movement, but the stimuli used by those investigators was quite different from that employed in the present studies.

A three-way and a four-way interaction were significant in Experiment 1, but a closer analysis of these effects revealed an inconsistent pattern of interactions, most of which were not interpretable. One systematic pattern that did emerge from these analyses involved the left target location when the targets moved to the left. It was determined that the $X Y$ monitor had an uneven glow in the lower left area that could have been perceived by darkadapted subjects. The result may have been a reference line on the left edge of the display against which movements of the left target could have been judged. A subsequent study conducted in this laboratory using the same target locations and movement speeds as those in Experiment 1 , with the defective monitor component replaced, found similar peripheral-field effects, but these higher order interactions were not significant.

It was predicted that peripheral-field movement would influence the direction in which a target at a particular location would be expected to move, and thus would influence sensitivity to movement detection, depending upon its direction of movement. If inputs from sensory systems are compared with expected inputs based on past experience, as postulated by sensory conflict theories (see McCauley, 1984), then internal estimates of sensory organ sensitivity and the interpretation of sensory output may depend upon the perceived environment within which the inputs were generated (Oman, 1982). For example, a person might reasonably expect a stationary target to the right of his/her perceived direction of travel to move farther to the right as he/she approached and passed the target. Thus, sensitivity to object motion under viewing conditions consistent with perceived self-motion may be a function of the expected motion of a stationary object.

Planned comparisons of means did not support the hypothesis. For each of the target locations in both experiments, the mean collapsed $d^{\prime}$ measure for movements in the direction that would have been expected from a radially expanding flow pattern was lower than the mean collapsed $d^{\prime}$ for movements in the other directions, but the differences were not significant.
The saccadic latency of eye movements toward the targets was measured in both experiments, and although the average latency was longer when movement was occurring in the peripheral visual field $(2.2 \mathrm{msec}$ in Experiment 1 and $4.1 \mathrm{msec}$ in Experiment 2), neither difference was significant.

Following the experiment, the subjects were asked whether they had experienced sensations of selfmovement. In each experiment, 12 of the 24 subjects reported vection when the movement occurred in the peripheral visual field. Analyses of the data using the report of vection as a between-subjects variable did not reveal any significant effects or interactions involving this factor in either the judgment or the saccadic latency data.

It appears that the process responsible for the peripheral field's effect on object-motion detection occurred both for subjects who reported vection and for those who did not. If visual-vestibular interaction is responsible for the differences in ability to detect object motion and for the sensations of self-movement, the fact that no differences were found between subjects reporting vection and those not reporting vection raises two possibilities with respect to the basis of the peripheral-field effect on object-motion detection. First, it may be that the moving peripheral-field condition did affect vestibular efference, which was the mediating influence on target motion detection, but that the effect was insufficient to produce a compelling sense of motion in some subjects. It is possible that cognitive factors related to the experimental context, such as the stationary chin- and headrest, suppressed the psychological experience of illusory self-movement, but not the physiological response of the vestibular system underlying the sensitivity decrease. Second, and more speculatively, it may be that subjects not reporting vection simply were not influenced by any significant interaction between the visual and vestibular systems. In this case a more direct interaction between the focal and ambient visual systems, without mediation through the vestibular system, could have been the basis for the observed changes in motion detection sensitivity.

\section{REFERENCES}

Büchele, W., Degner, D., \& Brandt, Th. (1980). Thresholds for object-motion perception raised by concurrent head-movements. Pflüger's Archiv: European Journal of Physiology, 384, R33, (Abstract 133).

Clark, B., Randle, R. J., \& Stewart, J. D. (1977). Vestibular-ocular accommodation reflex in man. Aviation, Space, \& Environmental Medicine, 46, 1336-1339.

Held, R., Dichgans, J., \& Bauer, J. (1975). Characteristics of moving visual scenes influencing spatial orientation. Vision Research, 15, 357-365.

Holm-JenSEN, S. (1982). Interference between synchronous optokinetic nystagmus and vestibular nystagmus. Acta Oto-Laryngologica, 93, 375-385.

JoNes, G. M. (1982, October). Adaptive control in the vistibulo-ocular system: Principles and mechanisms. Paper presented at the Symposium on the Neurophysiological Basis of Motor Disorders, Chicago, IL. 
Lishman, J. R., \& LEE, D. N. (1975). The autonomy of visual kinaesthesis. Perception, 2, 287-294.

Macmillian, N. A., \& KaPlan, H. L. (1985). Detection theory analysis of group data: Estimating sensitivity from average hit and falsealarm rates. Psychological Bulletin, 98, 185-199.

McCAuley, M. E. (Ed.). (1984). Research issues in simulator sickness: Proceedings of a workshop. Washington, DC: National Academy Press.

OMAN, C. M. (1982). A heuristic mathematical model for the dynamics of sensory conflict and motion sickness. Acta Oto-Laryngologica, Supplement 392.
Probst, T., KrafCZyK, S., Brandt, Th., \& Wist, E. (1984). Interaction between perceived self-motion and object-motion impairs vehicle guidance. Science, 225, 536-538.

Probst, T., \& Wist, E. R. (1982). Impairment of object motion perception during head movements. Perception, 11, A33.

Swets, J. A., \& Pickett, R. M. (1982). Evaluation of diagnostic systems: Methods from signal detection theory. New York: Academic Press.

(Manuscript received for publication August 24, 1987.) 